\title{
An incremental numerical method for calculation of residual stresses and strains in cold-formed steel members
}

\author{
H. Amouzegar ${ }^{\mathrm{a}}$, B.W. Schafer ${ }^{\mathrm{b}}$, M. Tootkaboni ${ }^{\mathrm{a}, *}$ \\ ${ }^{a}$ Department of Civil and Environmental Engineering, University of Massachusetts Dartmouth, 285 Old Westport Rd., North Dartmouth, MA \\ 02747-2300, USA \\ ${ }^{b}$ Department of Civil Engineering, Johns Hopkins University, Latrobe Hall 210, Baltimore, MD 21218, USA
}

\begin{abstract}
The residual stresses and strains in cold-formed steel members are a result of the manufacturing process. It has been shown that the variation of residual stresses through the thickness of cold-formed steel members is not linear. In this study a numerical algorithm is developed to calculate the through-thickness variation of residual stresses and strains. The algorithm calculates the stresses and strains by viewing the manufacturing process as a combination of elasto-plastic bending and springback in a wide plate under plane strain conditions. In order to calculate the plastic deformations, the Prandtl-Reuss flow rule associated with von Mises yield criterion is used. With regard to satisfying the boundary conditions on the surface, the bisection method is used to find the location of the neutral axis. The results obtained via the proposed algorithm are verified with the available closed formed solutions, finite element analysis results and experimental measurements. A parametric study is performed to evaluate the effect of the coil radius and cross-sectional and material properties on the residual stresses and strains. It is shown that, while in the corner regions the most important parameter is the corner radius, it is the coil radius and yield stress that play a significant role in the variation of residual stresses and strains in the flat regions.
\end{abstract}

Keywords: Cold-forming, Residual stress, Residual strain, Cold-formed steel members, Elasto-plastic bending

\section{Introduction}

Residual stresses in hot-rolled steel members, created due to uneven cooling after hot-rolling or welding, do not vary considerably through the thickness of the member $[1,2]$. In cold-formed steel members, residual stresses are generated as a result of the manufacturing processes i.e. coiling, uncoiling, flattening and cold-forming of the cross section (Fig. 1). In contrast to hot-rolled members, residual stresses due to cold-working vary significantly across the thickness $[2,3]$. The magnitude as well as cross-thickness variation of residual stresses may play an important role in

${ }^{*}$ Corresponding author

Email address: mtootkaboni@umassd. edu (M. Tootkaboni)

Preprint submitted to Thin-Walled Structures

April 8, 2016

(C) 2016. This manuscript version is made available under the Elsevier user license 
the mechanical response of structural members that are made by passing steel sheets through a cold forming process. In fact, recent studies (see e.g. [4]) show that the load carrying capacity of cold formed steel columns under bi-axial moments and axial load may change (up to 3\%) if residual stresses and strains are included in the nonlinear analysis providing more justification for why design strategies such as Direct Strength Method (DSM) need to include the cold forming effects in their strength predictions (see [5]). In addition, certain features of the mechanical response, such as early yielding on the faces of cold formed steel members or the extent of local buckling in cold formed steel tubes, are directly related to the through thickness variation of residual stresses and strains in these members $($ see $[2,6])$.

Efforts have been made to measure residual stresses at the surface of cold-formed steel members $[7,1,8]$. The through-thickness residual stresses, however, have often been assumed to vary linearly between the measured surface values. Nevertheless, examination of thicker plates $[9,10,11]$ and theoretical and numerical studies $[12,13,6,14$, $15,16]$ have shown that residual stresses in cold-formed steel members have a nonlinear distribution through the thickness. Ingvarsson [12] and Rondal [13] presented a plastic deformation-based incremental numerical algorithm to simulate the bending of a wide plate by using Prandtl-Reuss flow rule and von Mises yield criterion to predict residual stresses at the corners of the cold-formed sections. Kato and Aoki [6] used the same approach to model the plastic deformations created during coiling, flattening and cold-forming sequence to obtain residual stresses and strains in steel tubes. They ignored the normal stresses through the thickness, section thinning and the shifting of the neutral axis. Quach et al [14] developed an analytical solution to predict the residual stresses and strains due to coiling, uncoiling and flattening. They used Prandtl-Reuss flow rule and von Mises yield criterion to calculate the ratio of transverse stress to longitudinal stress across the thickness and assumed strains, from the coiling to the flattening process, are small enough to ignore strain hardening. A finite element model with plane strain elements was later used to simulate the press-braking procedure [15]. The model has been validated against experimentally measured residual stresses of press-braked thick plates $[9,10]$ and residual strains on the surfaces of lipped channel sections [1]. Moen et al [16] tried to simulate the manufacturing process starting from coiling. They proposed a simplified prediction method to estimate the residual stresses and strains through the thickness of cold-formed steel members. The prediction method was validated against experimental data. It was shown that for the flat parts of the lipped channels, the results are more or less compatible, however, the prediction method overestimated the longitudinal residual stresses at the corners.

This paper attempts to present a comprehensive incremental numerical algorithm to calculate the residual stresses and strains in cold-formed steel members. The algorithm is developed in such a way that it simulates the entire manufacturing process. The main part of the algorithm is solving the elasto-plastic bending problem in polar coordinates under plane strain conditions. It uses the associated Prandtl-Reuss flow rule with von Mises yield criterion to formu- 
late the relationships between plastic strain increments and total strain increments. Strain hardening, section thinning, shift of neutral axis and out-of-plane stresses are all considered. To demonstrate the performance and applicability of the proposed method, the results are compared with analytical predictions of stresses and strains in coiling and flattening, the results of finite element simulations of roll-forming and press-braking and the experimental measurements of residual stresses and strains in press-braked samples. A parametric study is also conducted on the effects of different parameters on the variability in the distribution of residual stresses and strains.

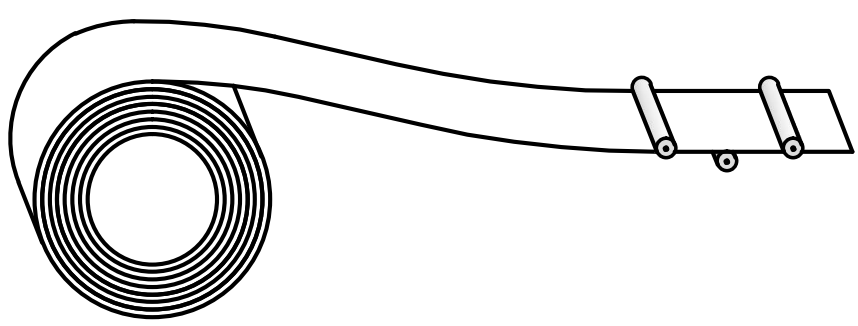

sheet steel coil - uncoiling and flattening

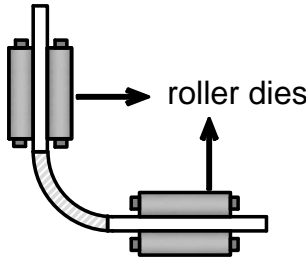

roll-forming

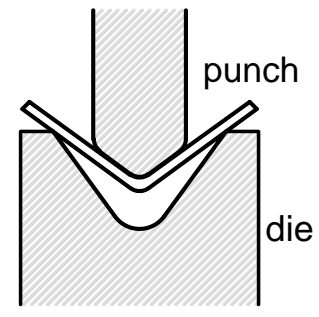

press-braking

Fig. 1: Manufacturing process for cold-formed steel members

\section{Coordinate system and nomenclature}

A polar coordinate system as illustrated in Fig. 2 is used where $\phi, \rho$ and $z$ denote longitudinal, radial and transverse directions respectively. The sign convention for stress and strain is positive for tension and negative for compression.
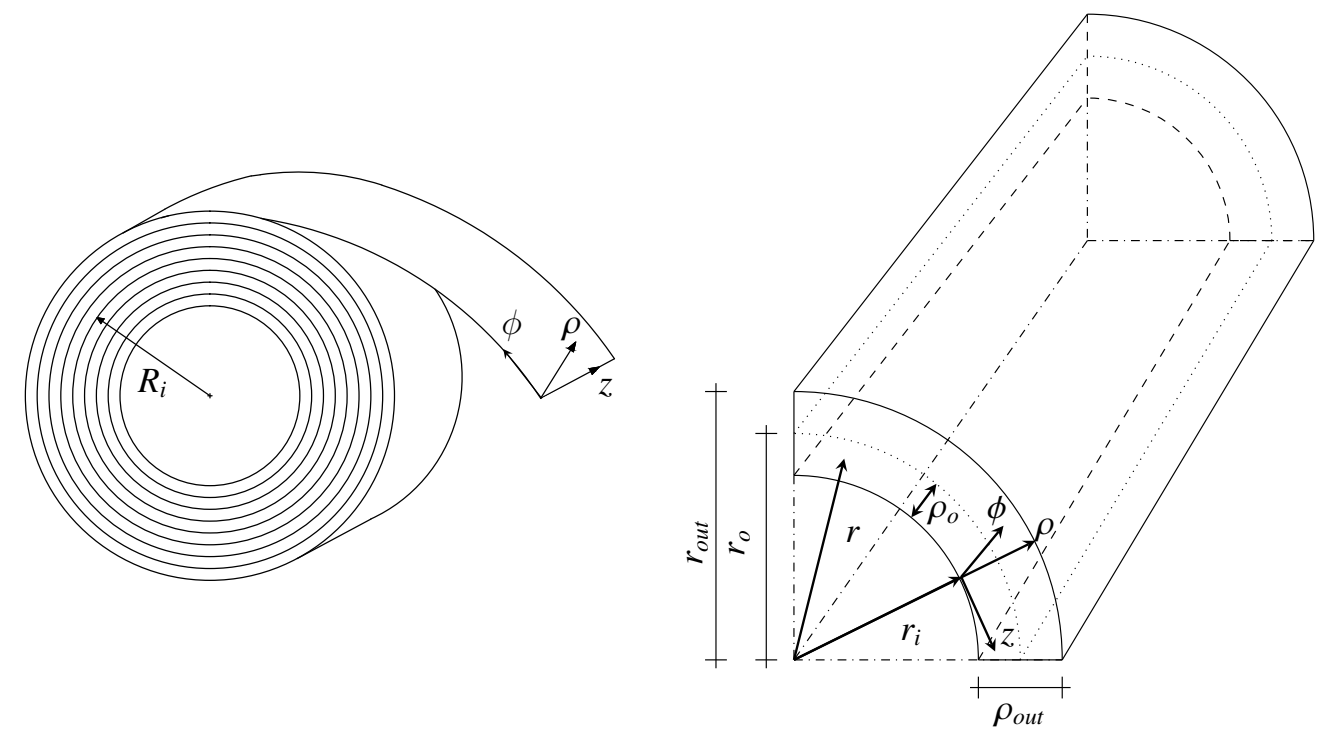

Fig. 2: Coordinate system 
The nomenclature adopted to represent different physical quantities throughout the manuscript is as follows:

$t$

$c$

$E$

$n_{c}, n_{t}$

$R_{i}$

$r, r_{i}$

$\rho$

$\rho_{o}, \rho_{\text {out }}$

$\epsilon_{\phi}, \epsilon_{r}, \epsilon_{z}$

$\epsilon_{\phi}^{e}, \epsilon_{r}^{e}, \epsilon_{z}^{e}$

$\epsilon_{\phi}^{p}, \epsilon_{r}^{p}, \epsilon_{z}^{p}$

$d \epsilon_{\phi}, d \epsilon_{r}, d \epsilon_{z}$

$d \epsilon_{\phi}^{e}, d \epsilon_{r}^{e}, d \epsilon_{z}^{e}$

$d \epsilon_{\phi}^{p}, d \epsilon_{r}^{p}, d \epsilon_{z}^{p}$

$\epsilon_{p}$

$\sigma_{\phi}, \sigma_{r}, \sigma_{z}$

$d \sigma_{\phi}, d \sigma_{r}, d \sigma_{z}$

$v$

$\sigma_{y}, \sigma_{e}$

$\sigma_{i}$

$H$
Thickness

Curvatures of inner surface

Young's modulus

Number of curvature increments and through-thickness segments respectively

Radius of sheet coil

Radial coordinate and inner radius respectively

Internal radial coordinate beginning at the inner surface

Distance from inner surface to neutral and outer surfaces respectively

Total strains in longitudinal, radial and transverse directions respectively

Elastic strains in longitudinal, radial and transverse directions respectively

Plastic strains in longitudinal, radial and transverse directions respectively

Total strain increments

Elastic strain increments

Plastic strain increments

Equivalent plastic strain

Stresses in longitudinal, radial and transverse directions respectively

Stress increments

Poisson's ratio

Yield stress from the uniaxial test and equivalent stress respectively

Radial pressure at inner surface

Strain hardening rate $\left(d \sigma_{e} / d \epsilon_{p}\right)$ in linear hardening

\section{Manufacturing process and the history of stresses}

It is assumed that the steel sheet is free of any residual stresses prior to coiling. Plastic deformations during the manufacturing process are thus assumed to be the only cause of residual stresses in cold-form steel members. Consequently, in order to predict residual stresses and strains, it is necessary to follow the manufacturing process step by step. Before being formed into its final shape, the steel sheet from which the cold-formed member is made may experience plastic deformations caused by coiling, uncoiling and flattening processes (simply referred to as the coiling-flattening process). Subsequently, the stresses and strains at the corners of the member's cross-section will 
change as a result of cold-forming process.

\subsection{Coiling-flattening process}

After rolling, steel strips are coiled for shipping to the plant. Depending on the radial position in the coil, the sheet might have some residual stresses through the thickness. In preparation for the cold-forming process, the sheet is uncoiled and thus unloaded elastically due to an elastic springback. The residual stresses still present after uncoiling will further change during the flattening process. Fig. 3a shows the stress path for the outer fiber of a steel sheet during the coiling-flattening process. Here, it is assumed that the stress-strain curve is elastic-perfectly plastic and the state of stress is considered to be $2 \mathrm{D}$ as $\sigma_{r}$ is zero on the outer surface. Line $O-E C$ shows the elastic path as the sheet undergoes coiling. As soon as the outer fiber reaches the point $E C$, it starts to deform plastically and continues on the $E C-P C$ path. This path is on the yield envelope, associated with the von Misses criterion in plane stress condition. During the uncoiling process, the elastic springback moves the stress state on a linear path $(P C-E F)$ parallel to the $O-E C$ line. Subsequently, the flattening process will update the stress state. The $E F-P F$ line shows the stress path when the sheet undergoes flattening and the outer fiber starts to deform plastically again. Point $U_{1}$ is the ultimate limit of the stresses that is approachable but not reachable [14].

\subsection{Cold-forming process}

Press-braking and roll-forming are the two forming techniques that are used to form the cross section of the cold-formed steel members (Fig. 1). During the cold-working, residual stresses caused during the coiling-flattening process are updated. The bending direction in cold-forming is perpendicular to the bending direction in coilingflattening. Fig. $3 \mathrm{~b}$ shows the continuation of the stress path for the outer fiber from point $P F$ in Fig. 3a, during the cold-forming process. It is assumed that the strain hardening is isotropic, therefore, the subsequent yield surfaces are isotropic expansions of the initial yield surface [17]. Curve $P F-U_{2}-U_{3}$ shows the loading path and line $U_{3}-E$ shows the elastic unloading (elastic springback) path. The loading path is asymptote to a line with a slope of 2 i.e. $O-U_{3}$. It can be seen that by continued deformation, the stress path will approach this line along which the bending stress $\left(\sigma_{z}\right)$ is twice the stress perpendicular to the bending direction $\left(\sigma_{\phi}\right)$. 

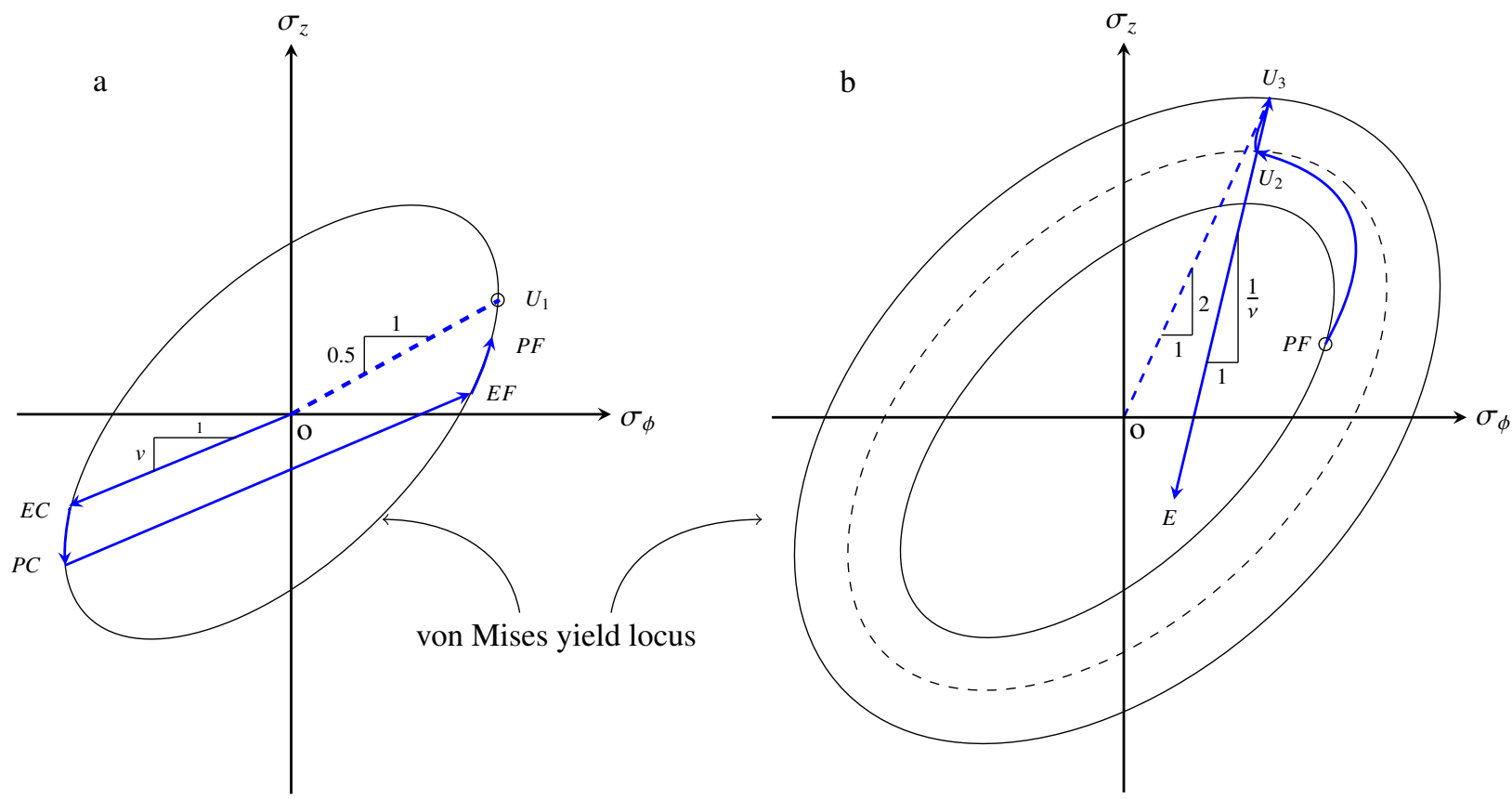

Fig. 3: Stress path for the outer fiber at the corner of a cold-formed section; (a) during coiling-flatting process, (b) during cold-forming process

\section{The incremental numerical method}

In what follows an incremental numerical method is proposed to calculate the residual stresses and strains in both the flat parts and corners of the cold-formed steel cross-sections, associated with the end of the coiling-flattening process and the end of the cold-forming process, respectively. The proposed approach simulates the cold-working process as the pure plastic bending of a wide plate in plane strain condition where the radius of curvature is increased incrementally.

\subsection{Governing equations}

Let $\phi$ represent the bending or longitudinal direction and $z$ and $r$ be the transverse and radial directions respectively. Due to symmetry, the local coordinate system in Fig. 2 coincides with the principal stress directions and there are no shear stresses and strains present. Also, due to the very high width to thickness ratio of the sheet, the lengthwise strain variations are ignored during cold-working. Therefore

$d \epsilon_{z}=0$ 
and

$\{d \epsilon\}=\left\{d \epsilon_{\phi}, d \epsilon_{r}, 0\right\}$

in which $d \epsilon_{\phi}, d \epsilon_{r}$ and $d \epsilon_{z}$ are the total strain increments. The total bending strain increment at any fiber with radial coordinate $\rho$ is given by

$d \epsilon_{\phi}=\frac{\rho-\rho_{o}}{\left(1+\rho_{o} c\right)^{2}} d c$

where $\rho_{o}$ is the distance to the neutral surface from the inner surface, $d c$ is the curvature increment at the inner surface and $c$ is the curvature of the inner surface given by

$c=\frac{1}{r_{i}}$

with $r_{i}$ is the radius of the curvature for inner surface. The total strain increment can be written as the sum of elastic and plastic parts as

$\{d \epsilon\}=\left\{d \epsilon^{e}\right\}+\left\{d \epsilon^{p}\right\}$

where $d \epsilon^{e}$ and $d \epsilon^{p}$ are the elastic and plastic strain increments respectively. The plastic part is derived from the associated flow rule (see [18]) and is written as

$\left\{d \epsilon^{p}\right\}=d \lambda \frac{\partial f}{\partial\{\sigma\}}$

where $d \lambda$ is a scalar factor of proportionality and $f$ is the yield function which for the case of von Mises yield criterion is expressed as

$f\left(\{\sigma\}, \sigma_{e}\right)=\frac{1}{2}\left\{\left(\sigma_{\phi}-\sigma_{r}\right)^{2}+\left(\sigma_{r}-\sigma_{z}\right)^{2}+\left(\sigma_{z}-\sigma_{\phi}\right)^{2}\right\}^{\frac{1}{2}}-\sigma_{e}=0$

in which $\sigma_{e}$ is the equivalent stress that can be expressed as a function of equivalent plastic strain $\epsilon_{p}$

$\sigma_{e}=\sigma_{e}\left(\epsilon_{p}\right)=k\left(\epsilon_{p}\right)^{n}$. 
The relationship between the equivalent stress and the equivalent plastic strain can be obtained from uniaxial experiments. For isotropic hardening (no Bauschinger effect) the yield function depends on equivalent plastic strain only through $\sigma_{e}$ and not explicitly (see [18]). The consistency condition then reads

$d f=\left\{\frac{\partial f}{\partial\{\sigma\}}\right\}^{T}\{d \sigma\}+\frac{\partial f}{\partial \sigma_{e}} d \sigma_{e}=0$

Now considering Hook's law in its differential form

$\{d \sigma\}=[C]\left\{d \epsilon^{e}\right\}$

which $[C]$ is the elastic constitutive matrix, and making use of Eq. (5) and Eq. (6) in consistency condition, the plastic multiplier, $d \lambda$, reads

$d \lambda=\frac{1}{h}\left\{\frac{\partial f}{\partial\{\sigma\}}\right\}^{T}[C]\{d \epsilon\}$

with

$h=\left\{\frac{\partial f}{\partial\{\sigma\}}\right\}^{T}[C]\left\{\frac{\partial f}{\partial\{\sigma\}}\right\}-\frac{\partial f}{\partial \sigma_{e}} \frac{d \sigma_{e}}{d \epsilon_{p}} \sqrt{\frac{2}{3}\left\{\frac{\partial f}{\partial\{\sigma\}}\right\}^{T}\left\{\frac{\partial f}{\partial\{\sigma\}}\right\}}$

and where the equivalent plastic strain has been defined as $d \epsilon_{p}=\sqrt{\frac{2}{3}\left\{d \epsilon^{p}\right\}^{T}\left\{d \epsilon^{p}\right\}}$ (see [19]). Multiplying both sides of Eq. (11) by $\partial f / \partial\{\sigma\}$ one arrives at the relationship between plastic strain increment and total strain increment as

$\left\{d \epsilon^{p}\right\}=\frac{1}{h}\left\{\frac{\partial f}{\partial\{\sigma\}}\right\}\left\{\frac{\partial f}{\partial\{\sigma\}}\right\}^{T}[C]\{d \epsilon\}$

By considering the equilibrium equations in the polar coordinate system and the fact that shear stresses are zero the radial stress increment can be written as

$d \sigma_{r}=\frac{\sigma_{\phi}-\sigma_{r}}{r} d r$ 
Integrating both side of Eq. (14) through the thickness and substituting from Eq. (4) leads to

$\sigma_{r}(\rho)=\int_{\rho_{\text {out }}}^{\rho} \frac{c}{1+c x}\left(\sigma_{r}-\sigma_{\phi}\right) d x$

where $\rho=r-r_{i}$ and at the boundary $\sigma_{r}\left(\rho_{\text {out }}\right)=0$. At the end of cold-forming, when the existing radial pressure or bending moment are balanced with internal plastic stresses the sheet will be elastically unloaded. The equations developed in [20] for elastic loading under inner pressure and pure bending in polar coordinate system are used to present unloading or elastic springback stresses associated with the loading condition depicted in Fig. 4.

For pressure unloading stresses we have

$\sigma_{r p}=\frac{r_{i}^{2} \sigma_{i}}{r_{\text {out }}-r_{i}}\left(1-\frac{r_{\text {out }}}{r}\right)$

$\sigma_{\phi p}=\frac{r_{i}^{2} \sigma_{i}}{r_{\text {out }}-r_{i}}\left(1+\frac{r_{\text {out }}}{r}\right)$

whereas for bending unloading stresses

$\sigma_{r b}=-\frac{4 M}{N}\left(\frac{r_{i}^{2} r_{\text {rout }}^{2}}{r^{2}} \log \frac{r_{\text {out }}}{r_{i}}+r_{\text {out }}^{2} \log \frac{r}{r_{\text {out }}}+r_{i}^{2} \log \frac{r_{i}}{r}\right)$

$\sigma_{\phi b}=-\frac{4 M}{N}\left(-\frac{r_{i}^{2} r_{\text {rout }}^{2}}{r^{2}} \log \frac{r_{\text {out }}}{r_{i}}+r_{\text {out }}^{2} \log \frac{r}{r_{\text {out }}}+r_{i}^{2} \log \frac{r_{i}}{r}+r_{\text {out }}^{2}-r_{i}^{2}\right)$

where $r_{i}$ and $r_{\text {out }}$ are the inner and outer radii respectively, $\sigma_{i}$ is the inner pressure, and

$N=\left(r_{\text {out }}^{2}-r_{i}^{2}\right)^{2}-4 r_{i}^{2} r_{\text {out }}^{2}\left(\log \frac{r_{\text {out }}}{r_{i}}\right)^{2}$

The bending moment, $M$, due to the existing longitudinal stresses $\left(\sigma_{\phi}\right)$ is given by

$M=-\int_{0}^{\rho_{\text {out }}} \sigma_{\phi}\left(\rho-\frac{\rho_{\text {out }}}{2}\right) d \rho$,

Strains in the transverse direction are zero, therefore, the stresses in the transverse direction due to pressure and bending unloading are respectively given by

$\sigma_{z p}=v\left(\sigma_{\phi p}+\sigma_{r p}\right)$ 
and

$\sigma_{z b}=\nu\left(\sigma_{\phi b}+\sigma_{r b}\right)$.

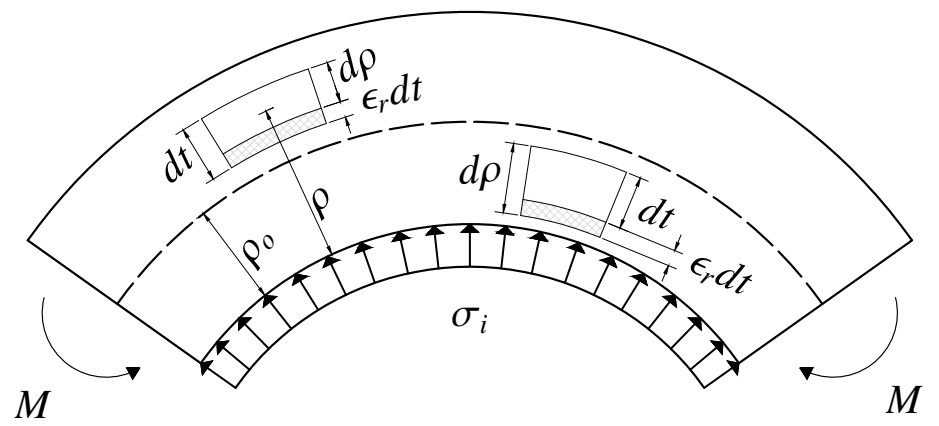

Fig. 4: Loading forces and section deformation

Furthermore, as is shown in Fig. 4, due to radial strains the thickness of a typical strip changes and we will have

$d \rho=\left(1+\epsilon_{r}\right) d t$

Integrating Eq. (24) results in

$\rho=\int d \rho=\int\left(1+\epsilon_{r}\right) d t$

After uncoiling, there might remain a curvature in the sheet due to the plastic deformations created in the coiling process. The radius of this curvature is calculated by

$R_{n}=\frac{1}{c_{1}-c_{2}}-\frac{\rho_{\text {out }}}{2}$,

in which

$c_{1}=\frac{1}{R_{i}+\rho_{\text {out }} / 2}$,

$c_{2}=\frac{\left(1-v^{2}\right) M_{\phi}}{E I}$, 
$M_{\phi}=-\int_{0}^{\rho_{\text {out }}} \sigma_{\phi b}\left(\rho-\frac{\rho_{\text {out }}}{2}\right) d \rho$

and

$I=\frac{\rho_{\text {out }}{ }^{3}}{12}+\rho_{\text {out }}\left(\frac{\rho_{\text {out }}}{2}-\rho_{o}\right)^{2}$

where $\rho_{o}, \rho_{\text {out }}, R_{i}$ and $\sigma_{\phi b}$ have been defined before.

\subsection{Numerical implementation}

In this section a numerical procedure is developed that uses equations in the preceding section to calculate the residual stresses and strains in each of the cold-working processes including coiling, uncoiling-flattening and coldforming. The deformation (curvature) is applied incrementally. The final curvature is divided into equal steps within which per-step stress and strain increments through the thickness are computed. In each step, curvature, stresses and strains are updated by calculating their cumulative sum. In the calculation of radial stresses using Eq. (15), the outer surface boundary condition is satisfied. It is assumed that the residual stresses and strains associated with the rollforming process are caused by pure bending, therefore, radial stress on the inner surface should be zero. On the other hand, when press-braking is used to form the steel sheet, the radial stress on the inner surface is equal to the pressure of the punch. From Eq. (15), radial stress at any radial coordinate is dependent on the distribution of radial and circumferential stresses through the thickness. In light of this argument, and considering the fact that in the bending of wide plates the neutral surface shifts towards the compression zone [21], a bisection procedure is used to adjust the location of the neutral surface in each step and to satisfy the boundary condition on the inner surface. The nonlinearity of the problem in the case of forming via press-braking makes it difficult to obtain the inner pressure at each step. Therefore, the same procedure is used to find the location of the neutral surface, that is, the inner pressure is ignored. The results in the next section show that this assumption in estimating the location of neutral surface compares very favorably with the results of the finite element analysis and experimental measurements of residual stresses due to the press-braking process. Finally, in plastically deformed regions of the thickness, the consistency condition has to be met, i.e.

$f\left(\{\sigma\}+\{d \sigma\}, \sigma_{e}\right)=f\left(\{\sigma\}, \sigma_{e}\right)=0$ 
Eq. (31) leads to (more details in [18])

$\{\sigma\}=\{\sigma\}+a\left\{\frac{\partial f}{\partial\{\sigma\}}\right\}$

where

$a=\frac{f\left(\{\sigma\}, \sigma_{e}\right)}{\left\{\frac{\partial f}{\partial\{\sigma\}}\right\}^{T}\left\{\frac{\partial f}{\partial\{\sigma\}}\right\}}$

The flowcharts in Figs. 5 and 6 depict different components of the proposed incremental numerical method. In the set of inputs, $n_{c}$ is the number of steps to reach the desired inner surface curvature,$R_{i}$ or $r_{i}$, starting from zero, $n_{t}$ is the number of segments trough the thickness, and $k$ and $n$ are the multiplication factor and exponent in Eq. (8). As can be seen in the bottom left corner of Fig. 5 a decision has to made on if the uncoiled and flattened sheet is fed to the cold-working process from bottom or top of the coil. This has to do with the dissimilarity between the stresses at the two sides of the neutral axis at the end of flattening process. In addition, there is a transition region between the two regions, the flat region and the corner region. However, results from the finite element modeling (see [15]) indicate these changes in the state of stresses in flat regions near the corner regions (after cold-forming) are negligible. Therefore in order to simplify the process these changes are often ignored in practical applications. Nonetheless, care should be given to the way the sheet has been fed in to the cold-forming process, and what the direction of bending in the corner region is. For example there is a different between the corner regions of a Z-section and a C-section cold-formed steel member when it comes to identifying the outer and inner surfaces. 


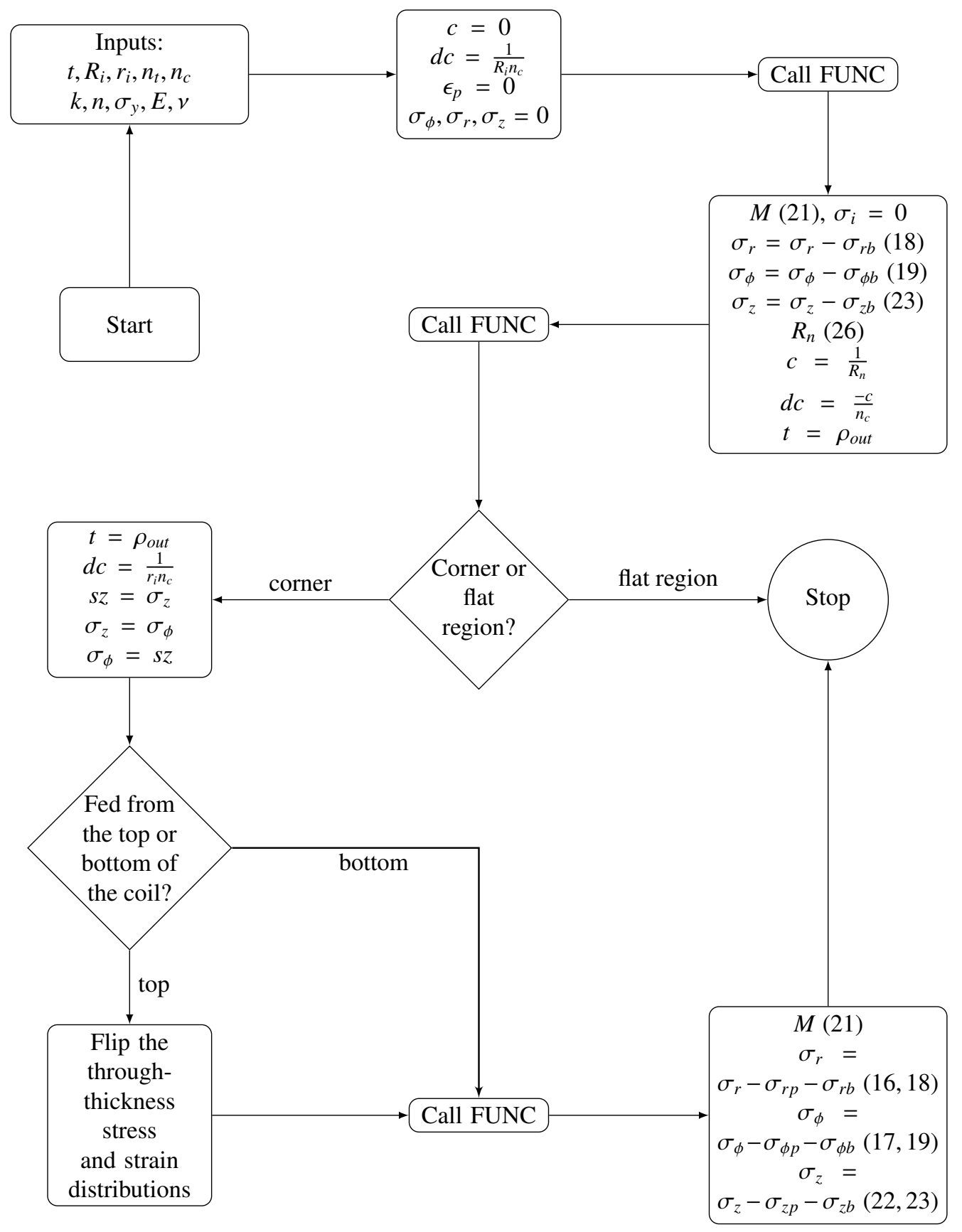

Fig. 5: Different components of the proposed incremental numerical method, numbers in parentheses denote Eq. numbers 


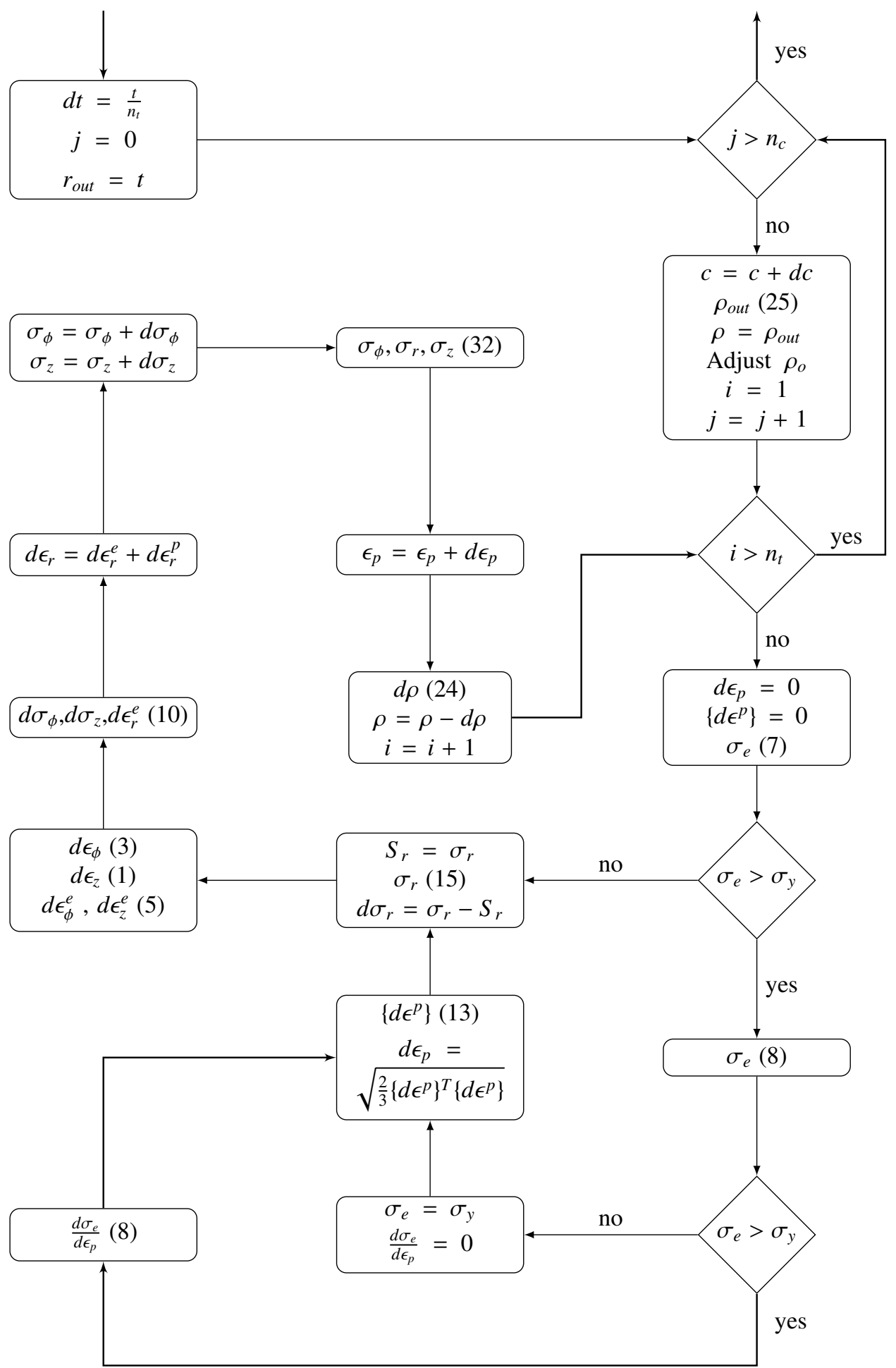

Fig. 6: Flowchart of the subroutine 'FUNC' in Fig. 5, numbers in parentheses denote Eq. numbers 


\section{Numerical results}

The incremental numerical method presented in the preceding section is compared with other references that predict or measure residual stresses through the thickness of cold-formed steel members. The comparison demonstrates the accuracy of the method in resembling closed form solutions as well as reproducing the results of the finite element simulation of plastic deformations. To further investigate the validity of the proposed method, a subset of results are compared with available experimental measurements.

\subsection{Comparison to analytical solution of coiling-flattening}

The closed-formed analytical solution presented in [14] predicts residual stresses and equivalent plastic strains from the coiling-flattening process. Transverse to longitudinal stress ratio is expressed as a function of the distance from the mid-plane and the material behavior is assumed to be elastic-perfectly plastic. Fig. 7 depicts the residual stresses and equivalent plastic strain through the thickness of a $2 \mathrm{~mm}$ plate with a coiling radius of $250 \mathrm{~mm}$ obtained via both the analytical solution of [14] and the proposed incremental numerical method (denoted by INM in the figures). The material properties used are $\sigma_{y}=250 \mathrm{MPa}, E=200 \mathrm{GPa}$ and $v=0.3$ and the results of the incremental numerical method are obtained in the absence of strain hardening and with $n_{t}$ and $n_{c}$ set to 50 and 1000 respectively. As shown in this figure, the results of the proposed incremental numerical method perfectly match the predictions of the analytical solution. 

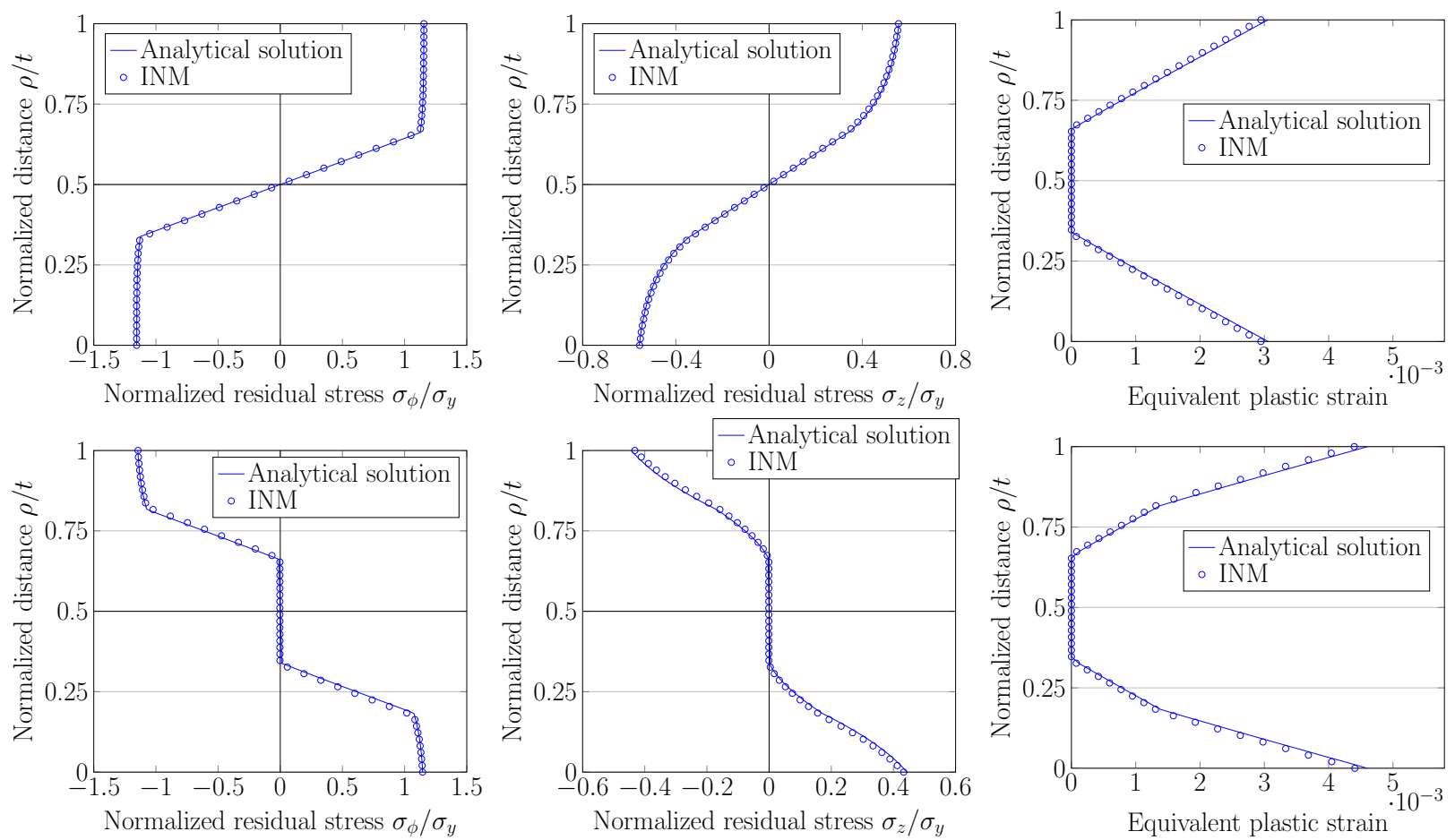

Fig. 7: Comparison of residual stresses and equivalent plastic strain between the incremental numerical method and analytical solution of [14]; top: after coiling, bottom: after flattening

\subsection{Comparison with the finite element analysis results}

In order to verify the accuracy of proposed incremental numerical method in modeling the cold-working of steel sheets, the roll-forming process is modeled in ABAQUS [22] as pure bending of a 2D strip under plane strain conditions. The strip is shown in Fig. 9. Its length and thickness are $150 \mathrm{~mm}$ and $25 \mathrm{~mm}$ respectively. The Young's modulus, yield stress and Poisson's ratio are chosen to be $200 \mathrm{GPa}, 500 \mathrm{MPa}$ and 0.3 respectively. The equivalent stress-equivalent plastic strain curve is shown in Fig. 8. All the nodes at the left edge are constrained in both the longitudinal and through the thickness directions. The node at the mid height of the right edge (free end) is defined as a reference node and the displacements of other nodes at this end are tied to it. To accurately estimate the distribution of stresses through the thickness, the thickness is divided into 25 plane strain elements; CPE4R elements in ABAQUS nomenclature. To resemble a realistic setting, it is assumed that the strip has already experienced the coiling-flattening deformations with a coiling radius of $1500 \mathrm{~mm}$. The reference node is rotated in order to create the stresses at a desired curvature $\left(r_{i}=100 \mathrm{~mm}\right)$. Fig. 10 and Fig. 11 show the comparison between the results of the incremental numerical method and the finite element simulations at an inner radius of $100 \mathrm{~mm}$ and after the folded sheet has gone through a elastic springback. A very close agreement in the equivalent plastic strains and stresses after loading and unloading is observed demonstrating the accuracy of the proposed framework. 


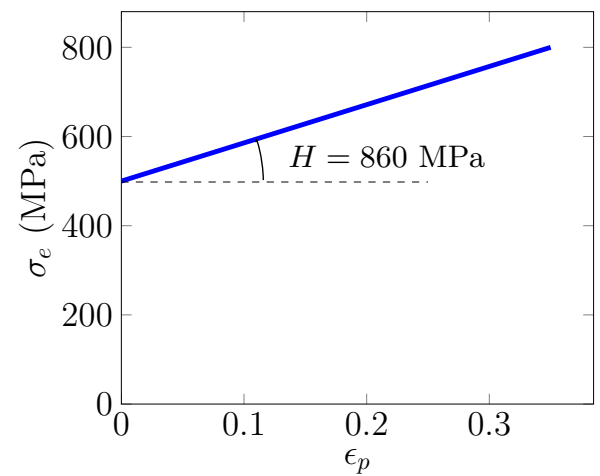

Fig. 8: Equivalent stress-equivalent plastic strain curve used in finite element modeling

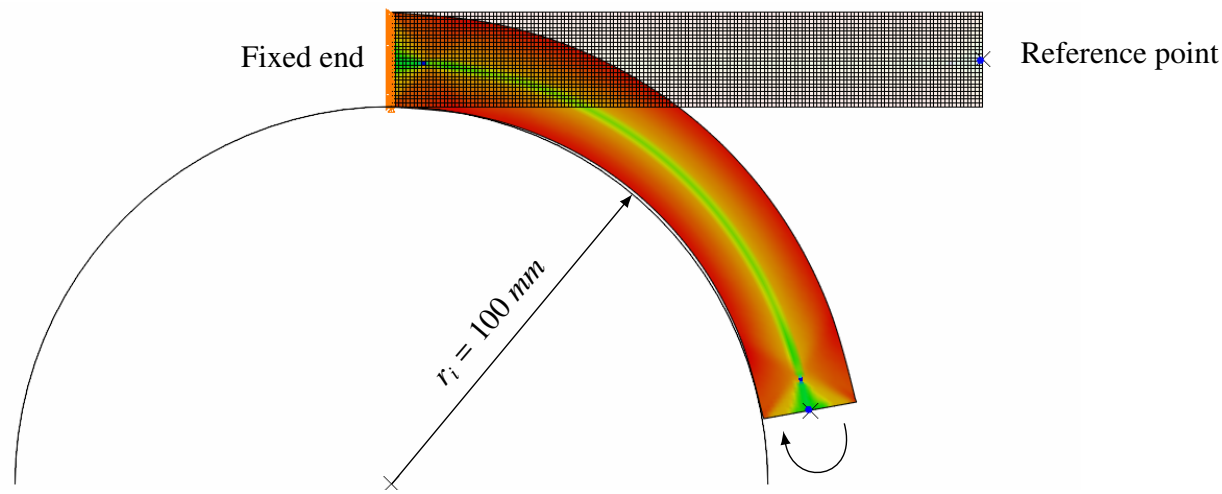

Fig. 9: Finite element model of a 2D strip under plane strain condition bent to a constant curvature 
(a)
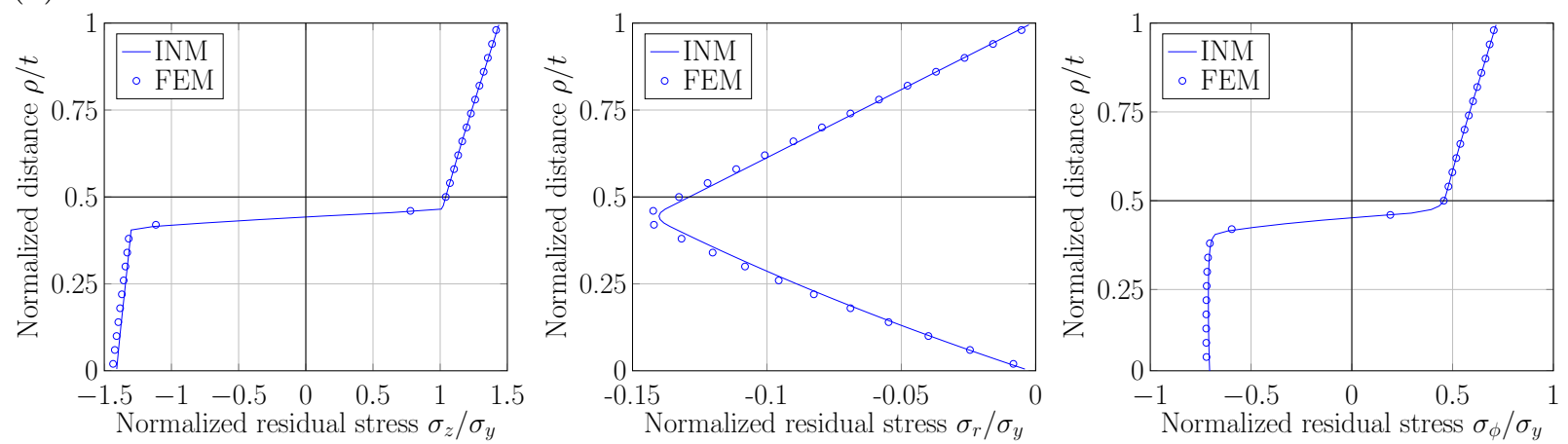

(b)
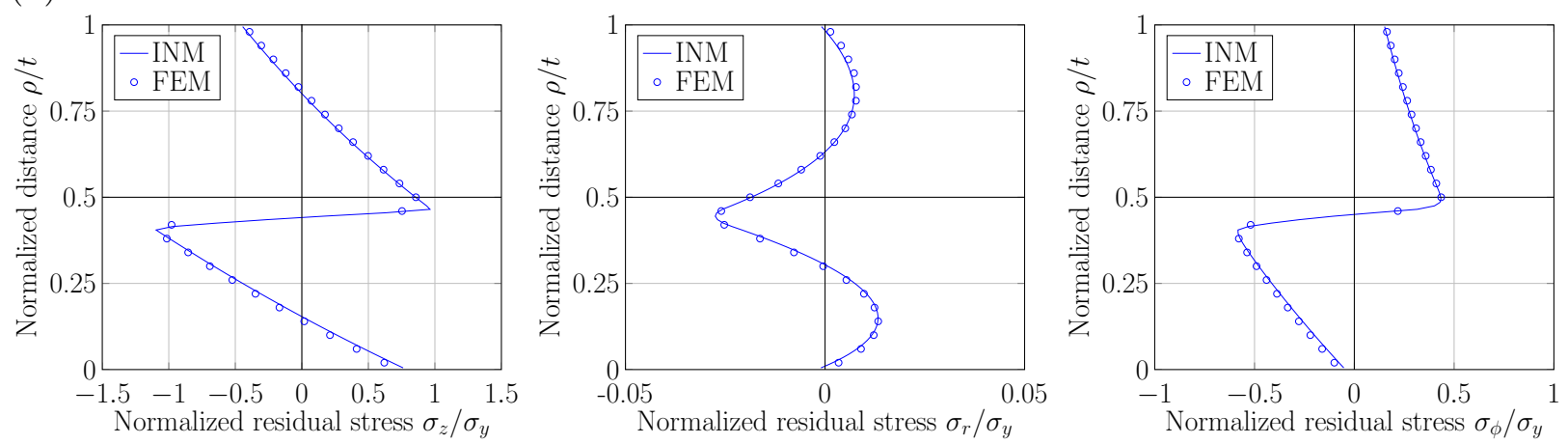

Fig. 10: Comparison of residual stresses between the incremental numerical method and the finite element method; (a) after loading, (b) after unloading

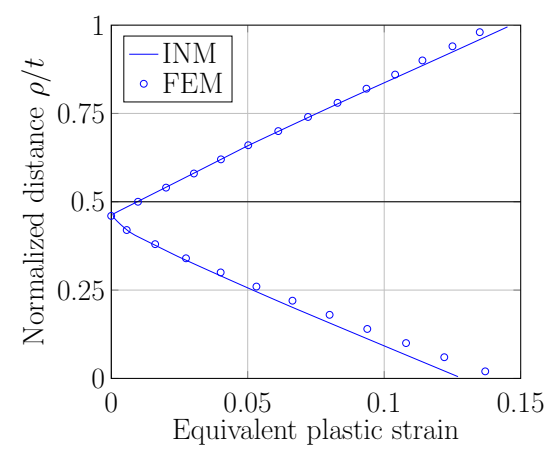

Fig. 11: Comparison of equivalent plastic strain between the incremental numerical method and the finite element method

Finite element modeling of cold-forming via press-braking has been performed by Quach et al. [15]. They verified their results with measured residual stresses and strains given in $[1,9,10]$. Both experimental measurements $[9,10]$, and the result of finite element simulations [15] as well as the results obtained from the proposed incremental numerical method for a 1 in thick HY-80 steel plates (see [10], see also Fig. 12a), four different bending radii (1.5, 2.5, 3.5 and 5.5 in.) and a bend angle of $90^{\circ}$ are presented in Fig. 13. The yield stress, the Young's modulus and 
the Poisson's ratio have been taken to be $\sigma_{y}=593 \mathrm{kN} / \mathrm{mm}^{2}, E=203.25 \mathrm{kN} / \mathrm{mm}^{2}$ and $v=0.28$ and the equivalent true stress-strain curve (Fig. 12a) has been used in the finite element modeling of press-braking process. As the results in Fig. 13 show, the finite element results and the results of incremental numerical method agree well with the experimental measurements. It is, however, noted that, overall, the incremental numerical method has predicted slightly larger transverse stresses at points near the neutral axis. This is, most probably, due to the approximations in satisfying the inner surface boundary condition when using the incremental numerical method to model the pressbraking process.

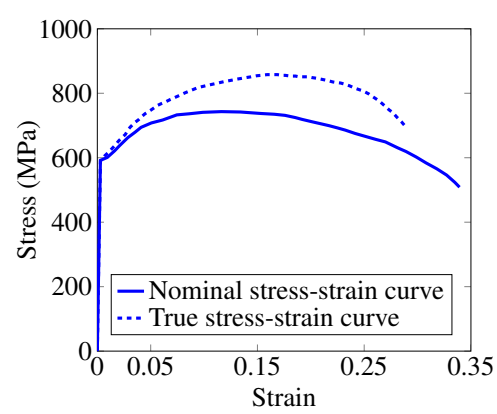

(a) HY-80 steel plate [10]

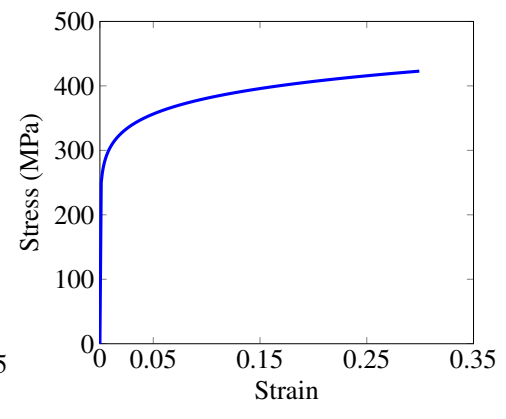

(b) PBC14 steel lipped channel [1]

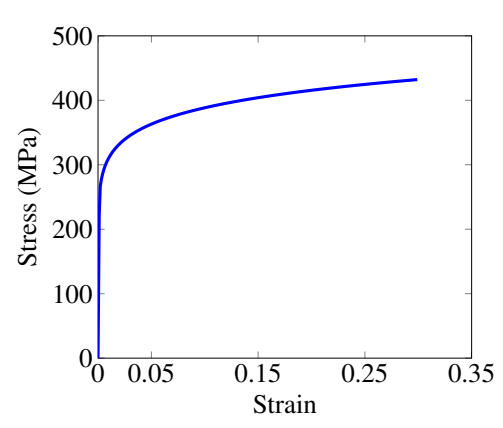

(c) P16 steel lipped channel [1]

Fig. 12: Stress-strain curves

Measured plastic strains on the surface of steel lipped channels caused as a result of press braking have also been reported in the literature. Fig. 14, for example, shows the measured longitudinal plastic strains on the surface of lipped channels PBC14 and P16 examined by Weng and Pekoz [1]. Finite element simulation results [15] and the results obtained from the incremental numerical method have also been included in this figure. The stress-strain curve for PBC14 and P16 are shown in Fig. 12 and the material properties, thickness and bending radii at the corner are provided in Table 1.

Table 1: Specifications of the lipped channels

\begin{tabular}{lcccc}
\hline Specimen & $t(\mathrm{~mm})$ & $r_{i}(\mathrm{~mm})$ & $\sigma_{y}(\mathrm{MPa})$ & $E(\mathrm{GPa})$ \\
\hline PBC14 & 1.80 & 3.96 & 250.1 & 203.3 \\
P16 & 1.63 & 2.39 & 220.9 & 202.7 \\
\hline
\end{tabular}

To initialize the state of stresses and plastic strain, that is to obtain the residual stresses and equivalent plastic strains through the thickness of the lipped channels PBC14 and P16 due to the coiling-flattening (and prior to pressbraking) Quach et al. [15] used the analytical solution developed in [14]. To determine the location of the strip in the coil (the coil radius) needed to use the relationships developed in [14] they used a trial and error process to fit the finite element results to the measured data from [1]. The obtained results were then used as the initial state in the 
finite element modeling of residual stresses and strains resulting from the press-braking simulation. The results are presented in Fig. 14 where results from the application of incremental numerical method with the same coil radius have also been included. As shown in this figure, including strain hardening in the calculation of the stresses and strains due to the coiling-flattening process in the incremental numerical method causes larger plastic strains in the flat parts of the lipped channels. Despite this difference, results of the incremental numerical method at the corners are in close agreement with the ones obtained from the finite element simulations. Note that the local strain peaks at the inner surface observed in finite element simulation results are due to local concentrations associated with the braking pressure. 

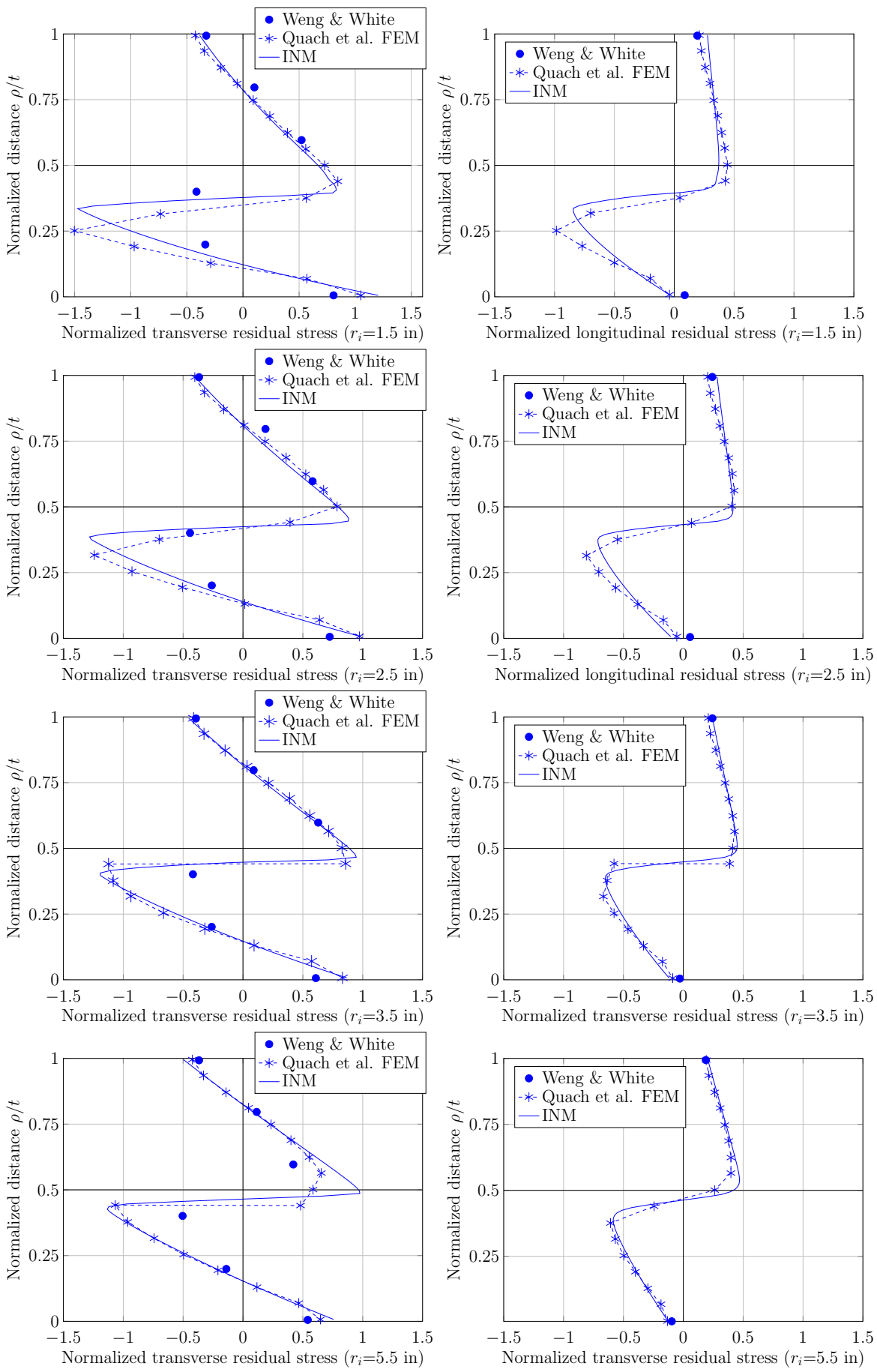

Fig. 13: Comparison of the results of incremental numerical method with finite element simulation results from Quach et al. [15] and experimental results from Weng and White $[9,10]$ 


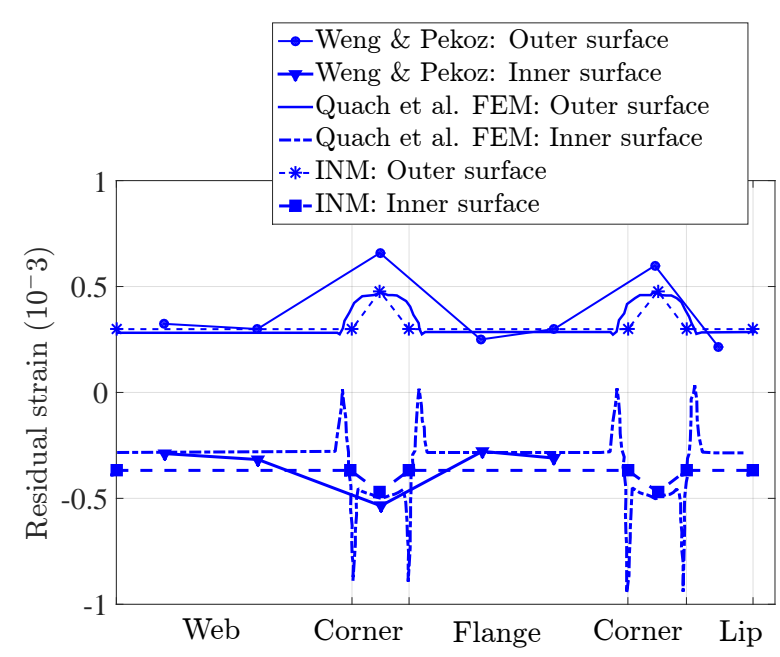

a) Specimen PBC14.

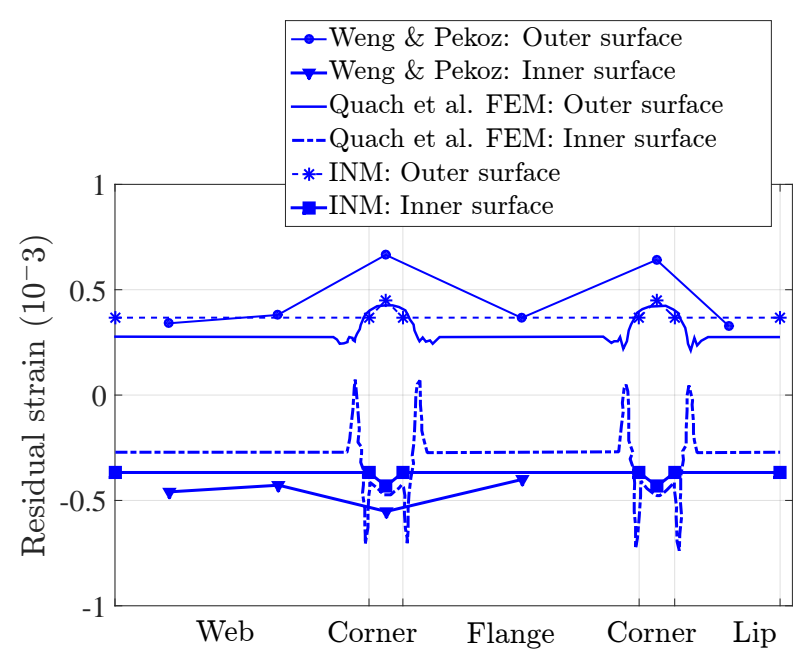

b) Specimen P16.

Fig. 14: Longitudinal surface residual strain distributions

\section{Parametric study}

In order to calculate the residual stresses and strains using the incremental numerical method, coil and corner radii, sheet thickness and material properties should be known. The ranges for thickness, corner radius and material properties of cold-formed steel members are given in $[16,23]$. Although, the coil radius is not known a priori, the ranges for inner and outer radii of coils prepared for shipping at a local roll-forming plant have been reported in [16] as 255 to $355 \mathrm{~mm}$ and 610 to $915 \mathrm{~mm}$ respectively. In this section a parametric study is conducted to examine the effect of variability in material properties, coil and corner radii and sheet thickness on the variation in residual stresses and strains in the flat and corner regions. The input parameters considered for this parametric study are shown in Table 2. The corner radii varies from $2 t$ to $8 t[16]$ and the ranges for thicknesses and yield stress in Table 2 are taken from 2012 IBC - SSMA product technical guide [23]. Variations in Young's modulus and the Poisson's ratio are ignored and they are assumed to be $203.3 \mathrm{GPa}$ and 0.3 respectively. The parameter $H$ in Table 2 represents strain hardening rate in the linear strain hardening model. Fig. 15 shows the normalized longitudinal and transverse residual stresses in the flat and corner regions. The residual stresses and strains are calculated for all combinations of the input parameters presented in Table 2 and it is assumed that the sheet is fed into the cold-forming process from the top of the coil, although, our numerical experiments show that the results are the same if the sheet is fed from the bottom. As can be seen in Figs. 15a and 15b in the corner regions, the ratios of the transverse and longitudinal residual stresses to the yield stress show little variations. This indicates that neither the initial state prior to cold-working, that is the residual stresses and strains due to the coiling-flattening process nor variation of the corner radius affect the normalized residual stresses after cold-forming severely. This is due to the effect of the elastic springback on final distribution of stresses. 
In fact variation of the stresses in the corner regions after cold-working and before the springback, are affected by the value of corner radius and variations of material properties (see Figs. 15c and 15d). However, elastic springback causes a reverse loading the value of which depends on the existing residual stresses. In other words, higher residual stresses cause more reverse loading due to springback. Therefore, regardless of the position of the sheet in the coil and the values of input parameters used in the cold-formed steel industry, one can estimate the transverse and longitudinal residual stresses in the corner regions of a cold-formed steel member by picking a representative curve built on the family of curves in Figs. 15a and 15b.

Table 2: Input parameters for the parametric study

\begin{tabular}{ccccccc}
\hline$t(m m)$ & $R_{i}(\mathrm{~mm})$ & $r_{i} / t$ & $\sigma_{y}(M P a)$ & $H(M P a)$ & $E(G p a)$ & $v$ \\
\hline 0.478 & 255 & 2 & 210 & 0 & 203.3 & 0.3 \\
0.719 & 350 & 4 & 345 & 406 & & \\
0.793 & 450 & 6 & 550 & 812 & & \\
0.879 & 550 & 8 & & & & \\
1.146 & 650 & & & & & \\
1.438 & 750 & & & & & \\
1.811 & 850 & & & & & \\
2.583 & 915 & & & & & \\
3.155 & & & & & & \\
\hline
\end{tabular}


a

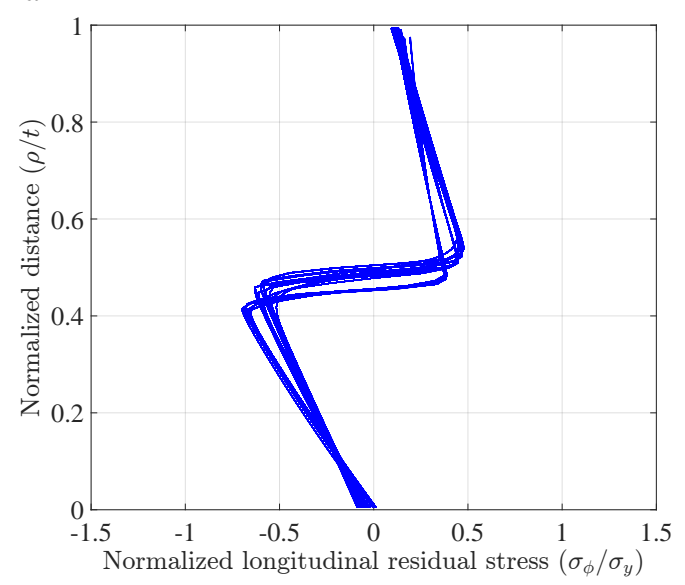

$\mathrm{c}$

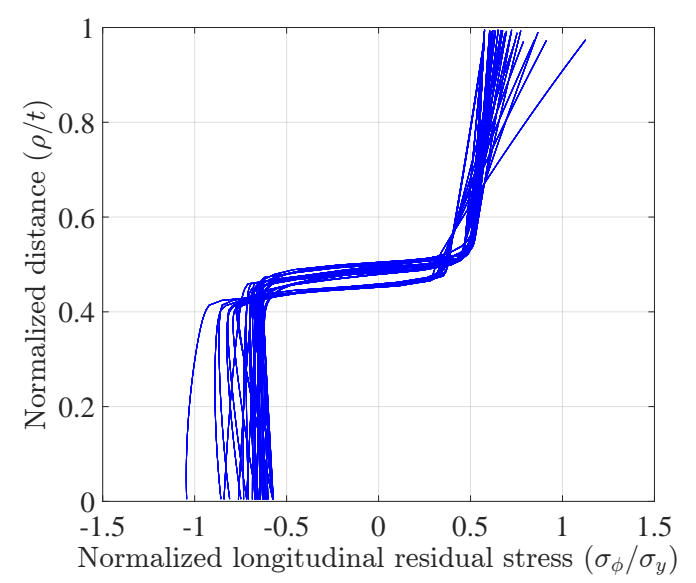

e

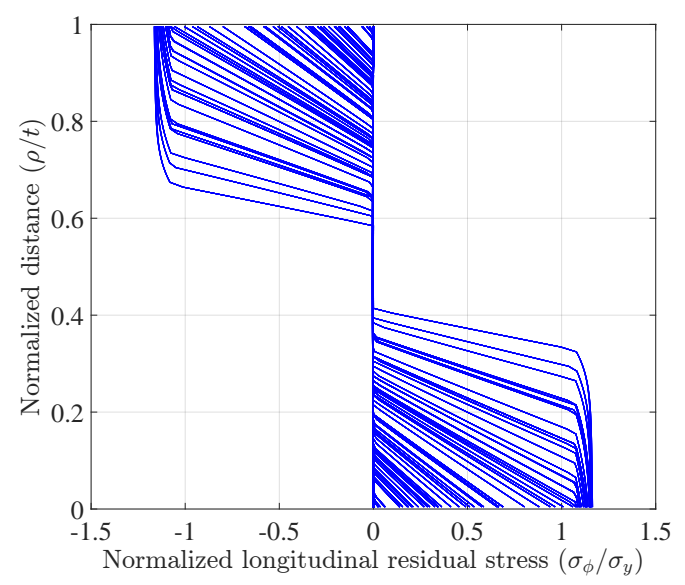

b

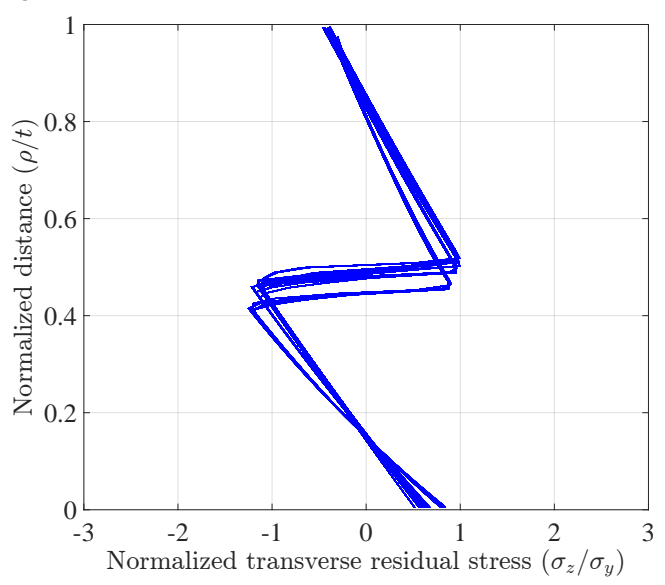

d

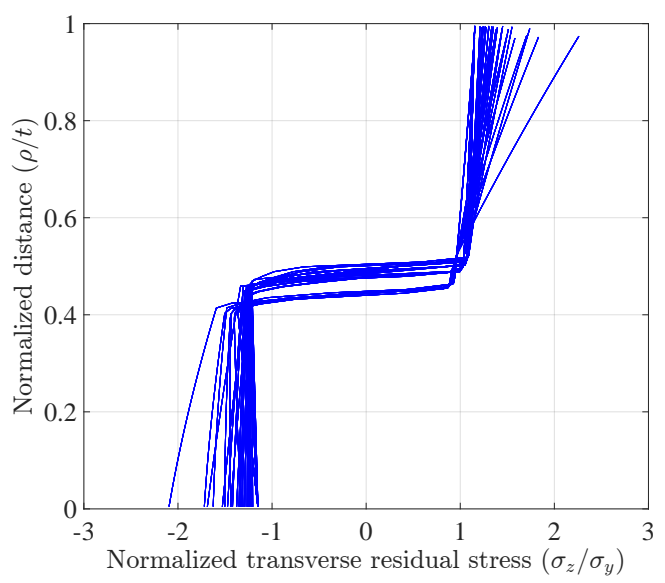

f

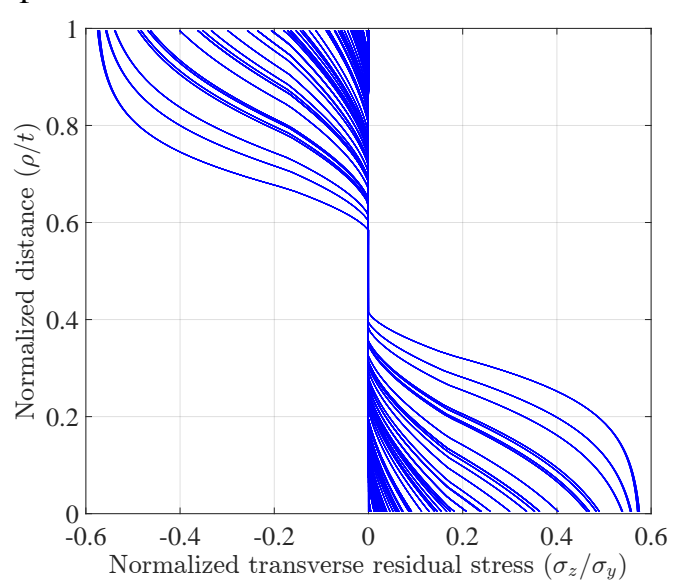

Fig. 15: Residual stresses for the combination of input parameters in Table 2; (a) \& (b) corner regions after springback, (c) \& (d) corner regions before springback, (e) \& (f) flat regions 
a

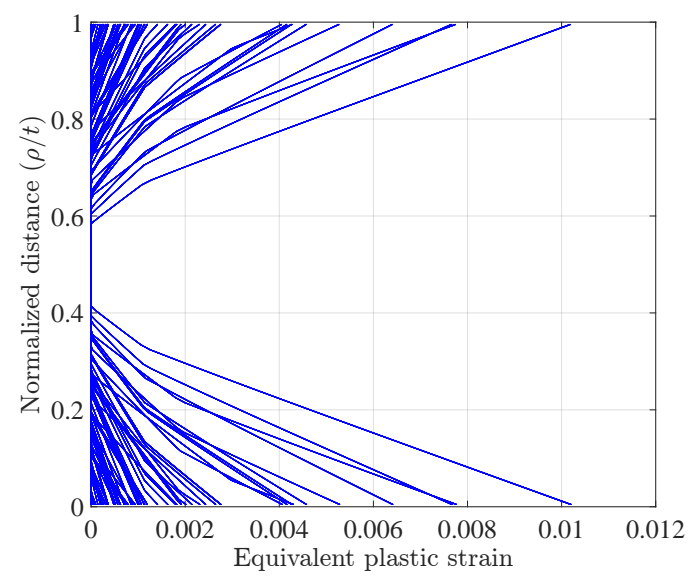

b

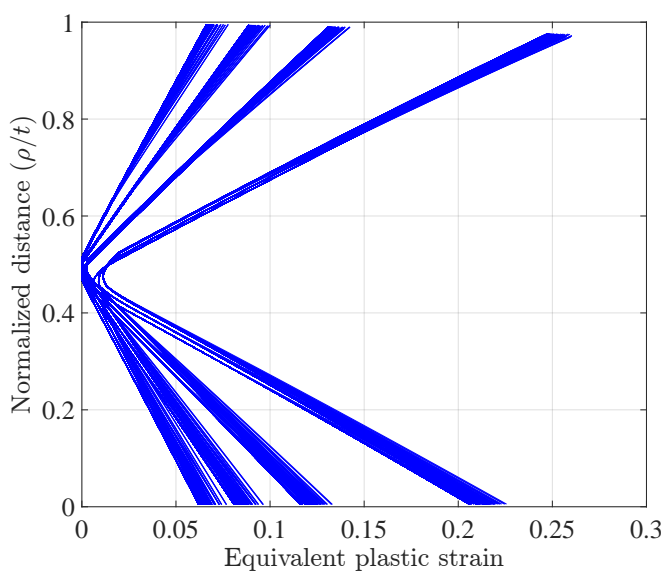

Fig. 16: Equivalent plastic strains for the combination of input parameters in Table 2 a) flat regions; b) corner regions

Residual stresses and strains in the flat regions show significant variation through the thickness as seen in Figs. 15e, $15 \mathrm{f}$ and 16a. There are four variables which can affect these variations: thickness, coil radius, yield stress and strain hardening rate. Fig. 17a shows the variation of the absolute maximum value of the longitudinal residual stress in the flat region versus thickness and coil radius for three different values of yield stress and a fixed value of strain hardening rate (the medium value in Table 2 or $406 \mathrm{Mpa}$ ). It can be seen that these three parameters significantly affect the formation of residual stresses in the flat regions. It can also be seen that, depending on the value of yield stress, there is a region in the thickness-coil radius plane where no residual stresses will develop. Fig. 17b shows the variation of the absolute maximum value of the normalized longitudinal residual stress in the flat region versus thickness and coil radius for three different values of strain hardening rate and a fixed value of yield stress (the maximum value in Table 2). As can be seen, the three surfaces associated with three different strain hardening rates are practically the same indicating the intangible effect of strain hardening on the variation of the residual stresses. This is because, as shown in Fig. 16a, the values of equivalent plastic strain in flat regions are very small. Therefore, it is expected that strain hardening will not have a significant effect on the residual stresses and strains in these regions. The ensemble of equivalent plastic strain curves for the corner regions shown in Fig. 16b presents four different groups. These groups correspond to the $r_{i} / t$ ratios shown in Table 2 and present significantly higher values of equivalent plastic strain when compared with those obtained for the flat region. Fig. 18 shows the influence of yield stress and sheet thickness on maximum longitudinal residual stress in the flat regions for different coil radii ranging from 255 to $915 \mathrm{~mm}$ (see Table 2) and a fixed value of $406 \mathrm{MPa}$ for strain hardening rate. As expected, increasing the coil radius reduces maximum residual stress. For yield stresses larger than $400 \mathrm{MPa}$ and a sheet thickness less than $2 \mathrm{~mm}$, the maximum residual stresses for coil radii of 850 and $915 \mathrm{~mm}$ are almost zero. Finally, Fig. 19 depicts the variations of section thinning 
and the maximum equivalent plastic strain in the corner regions versus $r_{i} / t$ for different combinations of thickness, coil radius, yield stress and strain hardening (represented by scatter bars). It is seen that, when compared with other input parameters, the corner radius has the most significant impact on the variability in maximum equivalent plastic strain and section thinning. Nevertheless, Fig 19b shows that in the most extreme scenario, the thickness is thinned less than $3 \%$.
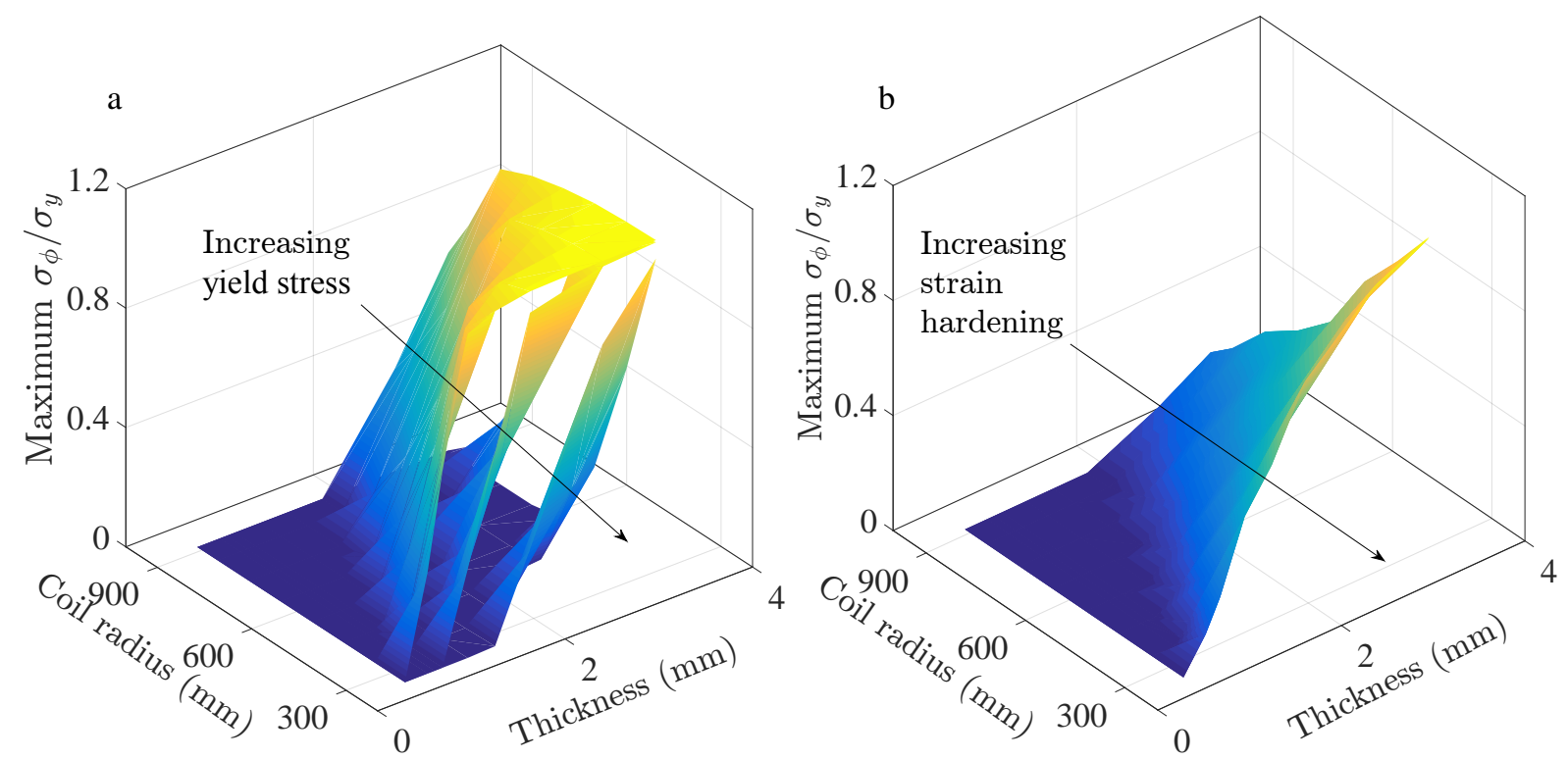

Fig. 17: Influence of sheet thickness and coil radius on maximum longitudinal residual stresses in the flat regions; (a) different yield stresses (b) different strain hardening rates 


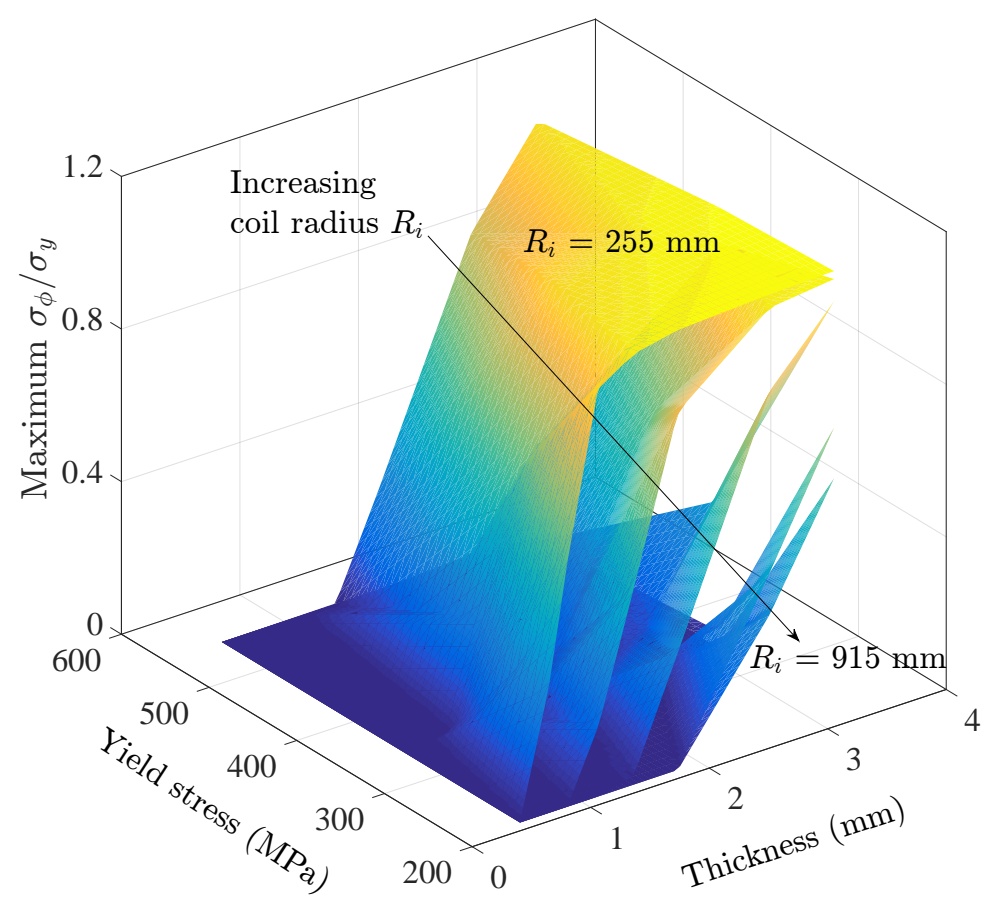

Fig. 18: Influence of sheet thickness and yield stress on maximum longitudinal residual stresses in the flat regions for different values of coil radii common in industry, and a fixed value of $406 \mathrm{MPa}$ for strain hardening rate
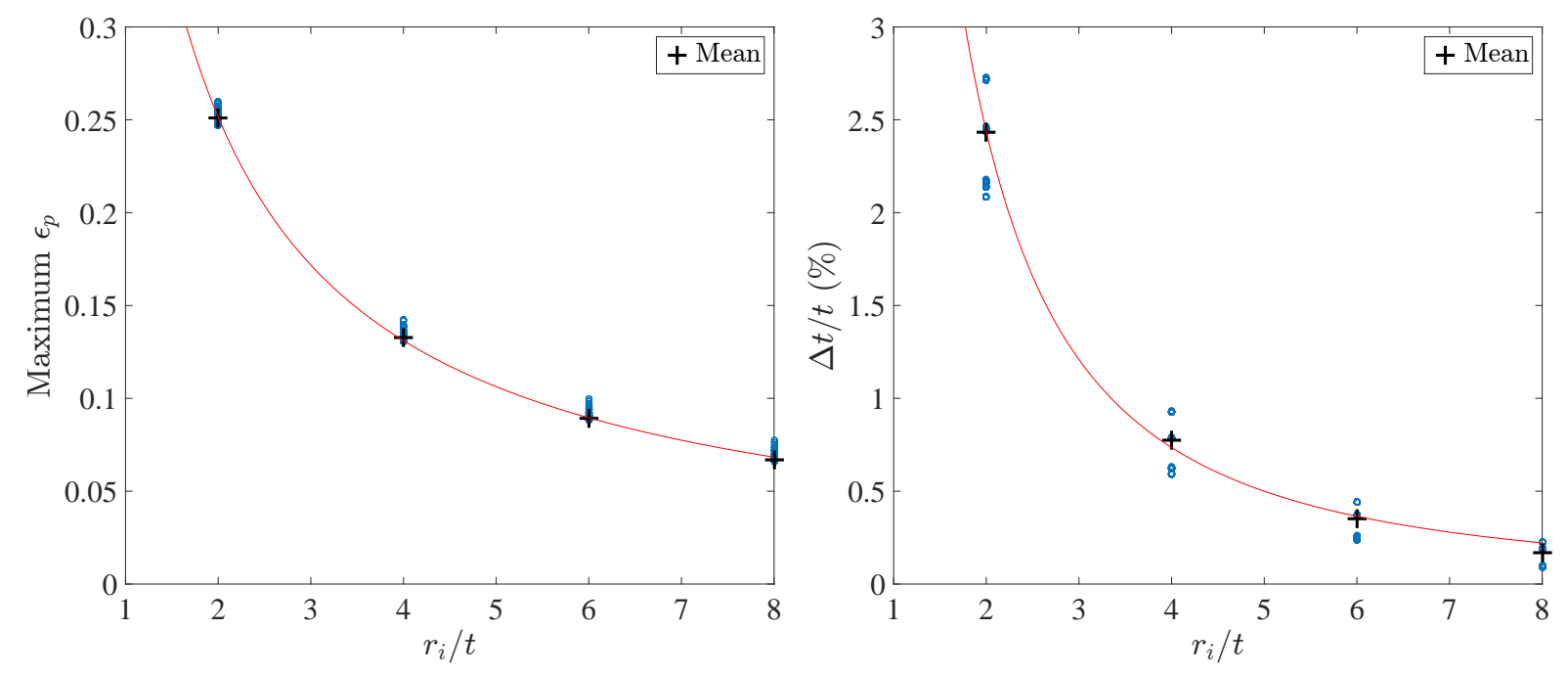

Fig. 19: Influence of corner radius on maximum equivalent plastic strain and section thinning; for a fixed value of $r_{i} / t$, circles correspond to combinations of input parameters in Table 2 


\section{Summery and conclusion}

An incremental numerical algorithm was developed to calculate the distribution of residual stresses and strains through the thickness of plates, channels or lipped channels that undergo the cold-working process. The cold-working process includes coiling, uncoiling, flattening and cold-forming operations such as roll-forming or press-braking. The associated Prandtl-Reuss flow rule with von Mises yield criterion was used to obtain the plastic strains increments based on the the total strains increments in the bending and radial directions. The strains perpendicular to the bending direction were ignored and it was assumed that strain hardening is isotropic. For residual stresses due to coiling, uncoiling and flattening, the algorithm was verified with existing analytical solutions. A finite element model was developed to simulate the roll-forming process where the stresses and strains that arise from the coiling-flattening process were considered as the initial state. The proposed incremental numerical method was then tested against the finite element analysis results indicating a high level of accuracy for the proposed framework. In order to validate the algorithm for modeling cold-forming through press-braking, its predictions were compared with the finite element analysis results already verified with the experimental measurements. It was shown that residual stresses and strains obtained from the incremental numerical method closely agree with both finite element and experimental results. A parametric study was conducted to examine the possible variations in residual stresses and strains. It was shown that residual stresses in the corner regions are marginally affected by the specifications of the cold-formed steel member. In the flat regions, however, depending on the coil radius, yield stress and the thickness of the sheet, significant residual stresses were formed. Strain hardening was showed to have a negligible contribution to the variability of residual stresses in the flat regions. It was also shown that, depending on the value of yield stress, no residual stresses will develop for particular values of sheet thickness and coil radius. Finally, the variations of section thinning and the equivalent plastic strain in the corner regions were shown to be highly affected by the corner radius.

\section{Acknowledgements}

This work was supported by National Science Foundation under Grants No. CMMI-1235238 and CMMI-1351742. This support is gratefully acknowledged.

\section{References}

[1] C. Weng, T. Peköz, Residual stresses in cold-formed steel members, Journal of Structural Engineering 116 (6) (1990) 1611-1625.

[2] B. Schafer, T. Peköz, Computational modeling of cold-formed steel: characterizing geometric imperfections and residual stresses, Journal of Constructional Steel Research 47 (3) (1998) 193-210.

[3] W. Yu, R. A. LaBoube, Cold-formed steel design, John Wiley \& Sons, 2010. 
[4] S. Torabian, B. Zheng, B. Schafer, Direct strength prediction of cold-formed steel beam-columns, Tech. rep., The Johns Hopkins University, Thin-Walled Structures Group (July 2014).

[5] S. Torabian, B. Zheng, B. Schafer, Direct strength prediction of cold-formed steel beam-columns, Tech. rep., The Johns Hopkins University, Thin-Walled Structures Group (July 2013).

[6] B. Kato, H. Aoki, Residual stresses in cold-formed tubes, The Journal of Strain Analysis for Engineering Design 13 (4) (1978) $193-204$.

[7] E. d. M. Batista, F. Rodrigues, Residual stress measurements on cold-formed profiles, Experimental Techniques 16 (5) (1992) 25-29.

[8] N. Abdel-Rahman, K. Sivakumaran, Material properties models for analysis of cold-formed steel members, Journal of Structural Engineering 123 (9) (1997) 1135-1143.

[9] C. Weng, R. White, Residual stresses in cold-bent thick steel plates, Journal of structural engineering 116 (1) (1990) 24-39.

[10] C. Weng, R. White, Cold-bending of thick high-strength steel plates, Journal of Structural Engineering 116 (1) (1990) 40-54.

[11] P. W. Key, G. J. Hancock, A theoretical investigation of the column behaviour of cold-formed square hollow sections, Thin-Walled Structures 16 (1) (1993) 31-64.

[12] L. Ingvarsson, Cold-forming residual stresses effect on buckling, in: Third specialty conference on cold-formed steel structures., Missouri S\&T (formerly the University of Missouri-Rolla), 1975.

[13] J. Rondal, Residual stresses in cold-rolled profiles, Construction and Building Materials 1 (3) (1987) 150-164.

[14] W. Quach, J. Teng, K. Chung, Residual stresses in steel sheets due to coiling and uncoiling: a closed-form analytical solution, Engineering structures 26 (9) (2004) 1249-1259.

[15] W. Quach, J. Teng, K. Chung, Finite element predictions of residual stresses in press-braked thin-walled steel sections, Engineering structures 28 (11) (2006) 1609-1619.

[16] C. D. Moen, T. Igusa, B. Schafer, Prediction of residual stresses and strains in cold-formed steel members, Thin-walled structures 46 (11) (2008) 1274-1289.

[17] H. C. Wu, Continuum mechanics and plasticity, CRC Press, 2004.

[18] W. F. Chen, D.-J. Han, Plasticity for structural engineers, Springer, New York, 1988.

[19] C. Berg, Note on construction of the equivalent plastic strain increment, J. Res., Natl. Bur. Stand. C 76 (1) (1973) $53-54$.

[20] S. Timoshenko, Theory of elasticity, Engineering societies monographs, McGraw-Hill, 1951.

[21] R. Hill, The Mathematical Theory of Plasticity, The Oxford engineering science series, Clarendon Press, 1983.

[22] ABAQUS, Abaqus 6.13 user's manual, Dassault Systems, Providence, RI, 2013.

[23] The steel stud manufacturers association (ssma). http://www.ssma.com. 